\title{
A Take-Home Message: Spillovers from Workplace Food Waste Campaigns to the Home
}

\section{Feiyang Wang}

LSE

Ganga Shreedhar ( $\nabla$ g.s.shreedhar@lse.ac.uk)

London School of Economics and Political Science

Matteo Galizzi

LSE

Susana Mourato

LSE

Article

Keywords: workplace food waste campaigns, human behaviours, food waste reduction

Posted Date: September 14th, 2021

DOl: https://doi.org/10.21203/rs.3.rs-903571/v1

License: (c) (i) This work is licensed under a Creative Commons Attribution 4.0 International License.

Read Full License 


\section{Abstract}

Understanding how workplace food waste campaigns impact behaviours at home can help promote broader lifestyle change than piece-meal behavioural interventions. Using a quasi-experimental field study and a survey of workers in the hospitality sector, we quantify the worker's responses to proenvironmental food waste campaigns. We find evidence that efforts to reduce food waste at work is associated with food saving actions at home, pointing to positive spillovers across these contexts.

\section{Main Text}

Behaviour change interventions aiming at reducing food waste, for example through smart technologies, information feedback and nudges, are crucial to address climate change, sustainable resource use, and food security ${ }^{1-5}$. Yet there have been few attempts to systematically test the effectiveness of such interventions ${ }^{1,3,6}$. In particular, there is little evidence about contextual spillovers, which occur when interventions changing behaviour on one context (e.g. a workplace campaign reducing food waste at a staff canteen) impacts behaviour in another context (e.g. less food wasted at home) ${ }^{7}$. Understanding whether impact of interventions can scale up across contexts is crucial to map the net effect of interventions and to pick those which inspire larger and more sustainable behavioural and lifestyle changes ${ }^{8}$.

Changing food waste behaviours across contexts is challenging for several reasons. The amount of food wasted by organisations and individuals is often unquantified and difficult to accurately measure, and its impact in terms of carbon emissions, for example, is frequently ignored or underestimated ${ }^{9-11}$. Moreover, various psychological processes may lead to positive or negative spillovers, whereby saving food at work can increase or decrease the likelihood of doing so at home. Positive spillovers may be more likely if the intervention affects intrinsic motivations to reduce food waste, for example by making salient environmental or resource wastage, or by priming environmental identity ${ }^{8,12}$. Negative spillovers may result if moral licensing occurs, such that people feel entitled to waste food at home because they believe they have already done their part at work ${ }^{13}$. Interventions aiming at highlighting the environmental impact of food behaviours are widely advocated to achieve positive spillovers, although there is limited evidence for this $5,11,8,13-15$. To the best of our knowledge, our study is the first to test if a behavioural intervention to reduce food waste at workplace spillovers to food behaviour at home.

The intervention we consider combined smart bins which automatically measure food waste with an information campaign using feedback in terms of the resource and environmental impacts of food waste. It was conducted in three hotels in Macau between the end of 2020 and the 2021 summer. In particular, we tracked the staff canteens of three hotels (A, B and C) and 1,536,610 meals in total, to examine impacts on employees' food waste reduction at work and at home during six months. To measure the effects of food waste campaigns in the workplace, we employed a difference-in-difference (DID) design where, in all the canteens, we gave fortnightly feedback on the reduction of food waste in 
each site as compared to the baseline period, while we also introduced some further interventions in some sites. The design of the study over the 5 rounds is summarised in Fig. 1 below. Basically, in control site $A$, we gave no feedback in the first two rounds, and we only provided food waste feedback in rounds 3-5. In treatment site B, food waste feedback was given for all the 5 rounds, while additional posters about the environmental benefits of reducing food waste were added in rounds $3-5$. The same food waste feedback and environmental messages were also given to treatment site $\mathrm{C}$ but images (e.g., food, trees, the globe) in the posters were anthropomorphised, since research shows that anthropomorphic cues could boost compliance with social causes and decrease food waste ${ }^{16-18}$.

Our study period was from $21 / 12 / 2020$ to $23 / 05 / 2021$, and the food waste feedback campaign ran between 09/02/2021 and 19/04/2021 in site B \& C, and between 09/03/2021 and 19/04/2021 in site A, with the baseline periods being 03/02/2021 to $16 / 02 / 2021$ for control site $A$, and $21 / 12 / 2021$ to 03/01/2021 for treatment sites B and C (see SI pp.6-8 for details). We had 7 weeks of data before any feedback posters were introduced, and we also tracked changes in food waste data for 5 further weeks after the posters were removed. Our outcome variable was grams of food waste per cover per day, automatically captured by the Winnow Sense (WS) system through smart bins in each site, which had the advantage of minimizing the operational challenges in manual weighing.

Insert Fig. 1 here.

The food waste at the control site A gradually increased throughout the study period and increased more rapidly after we removed the last round of feedback (Fig. 1). At treatment site B, food waste remained at the same level before and during the campaign period and increased slightly after the posters were removed. In contrast, food waste at treatment site $\mathrm{C}$ showed a reduction during the campaign period and kept decreasing after the posters were removed.

To eliminate the influence of unobserved fixed factors specific to the three canteens which can impact food waste (e.g., canteen size and layout), we used DID regression analyses to compare changes in food waste, rather than levels of food waste. During the food waste campaign, there were significantly more reductions in food waste at sites $B$ and $C$ as compared to the control site $A$ (DID site $B$ - site $A: \beta=-8.92$, $S E=4.65, z=-1.92, p=0.055,95 \% C l=[-18.05,0.20] ; D I D$ site $C-$ site $A: \beta=-17.49, S E=4.65, z=-3.76$, $p<0.001,95 \% \mathrm{Cl}=[-26.61,-8.37]$; see Methods and SI Table S2). These results suggest that exposure to environmental messages, especially the anthropomorphised ones, was associated with a significant decrease in food waste behaviors in the workplace.

To investigate if there were work-to-home spillovers, we relied on questionnaire data collected through an online survey with employees from all sites after the food waste campaign ended. The survey was built on Qualtrics and distributed during 26/04/2021 to 10/05/2021 via QR codes. To ensure clarity and comprehension, in each survey the questions and responses were available in both Chinese and English. The survey measured people's efforts to reduce food waste at home using four items (e.g., "Tried to reduce food waste at home", "Threw away less food") which we averaged to form a composite score. We 
also measured self-reported food waste reduction at work (efforts at work 1: "Had a conversation with work colleagues about food waste", efforts at work 2: "Tried to reduce food waste at work"), and psychological mechanisms like food waste motivations and beliefs, and environmental identity, which have been documented to influence both food waste behaviours and contextual spillovers in past studies $^{8,12,13,19-21}$. We also collected socio-demographic characteristics like age, gender, and income (see Methods and SI). We collected 1,253 responses, of which 1,198 were valid (see SI p.9). We analyzed responses from employees at all sites (rather than just the treatment sites) since all sites received food waste feedback, and since employees across sites could have taken different efforts to reduce their waste. Consistent with the actual food waste data, respondents from treatment site $C$ reported significantly more actions to reduce food waste at work than those from the control site, and this held even controlling for age, gender, education, income, motivations to save food, and environmental identity. The differences in self-reported food-saving actions at work between treatment site $B$ and the control site A were not statistically significant (see SI Table S7).

Regression analyses revealed that efforts to reduce food waste at work were positively and significantly associated with efforts to reduce food waste at home (efforts at work 1 : $\beta=0.23, S E=0.02, t=12.23, p<$ $0.001,95 \% \mathrm{Cl}=[0.19,0.27]$; efforts at work $2: \beta=0.39, S E=0.02, t=17.51, p<0.001,95 \% \mathrm{Cl}=[0.34,0.43])$. The estimated food waste reductions at home were statistically significant even controlling for site fixed effects, age, gender, education, income, motivations to save food, environmental identity, catalyzing beliefs, and compensatory beliefs (Fig. 2, also see SI Table S4). These results indicate that there were positive spillovers on efforts to reduce food waste from workplace campaigns to the home. In addition, echoing findings from previous research ${ }^{11}$, the catalyzing beliefs (e.g., "Being environmentally friendly is not about taking small actions; it is a complete approach to life.") predicted higher efforts to reduce food waste at home, while compensatory beliefs (e.g., "The environmental impact of wasting food at home can be made up for by saving food at work.") resulted in lower efforts (catalyzing beliefs: $\beta=0.07, S E=$ $0.040, t=2.06, p=0.04,95 \% \mathrm{Cl}=[0.004,0.145]$; compensatory beliefs: $\beta=-0.03, S E=0.01, t=-2.49, p=$ $0.013,95 \% \mathrm{Cl}=[-0.06,-0.01])$. Although money concerns seemed to motivate food saving at home more effectively than any moral or environmental concerns (motivation $1: \beta=0.08, S E=0.03, t=2.43, p=0.015$, $95 \% \mathrm{Cl}=[0.02,0.15]$ ), environmental identity (e.g., "I think of myself as an environmentally-friendly person.") was a no less important factor that positively predicted food waste reduction (environmental identity: $\beta=0.15, S E=0.03, t=5.04, p<0.001,95 \% \mathrm{Cl}=[0.09,0.21])$.

Insert Fig. 2 here.

Our study has several strengths. We quantified actual food waste over 1,536,610 meals in three hotel staff canteens in Macau over six months from a behavioural campaign at the workplace combining smart bins measurement and information feedback. In total, the three sites reduced approximately 9819.73 kilograms of food waste during the campaign as compared to the baselines, equivalent to 24,549 meals saved or 42.22 metric tons of reduction in $\mathrm{CO}_{2}$ emissions. We also examined self-reported food waste behaviours and motivations at home amongst the same employees. A recent review suggests only 17 articles feature behavioural interventions resulting in food waste reduction (all barring one were in 
American or European countries, $40 \%$ solely relied on self-reports or pictorial analyses of food waste, and typically focused on small groups) and no studies to date has considered behavioural spillover effects ${ }^{6}$. We innovatively contribute to this literature by providing evidence that workplace campaigns, and personal efforts to reduce food waste at work, are associated to food waste reduction also at home. Such positive contextual spillovers are an underattended yet important aspect of behavioural interventions in this area, as is designing interventions that scale impacts. In line with past studies ${ }^{17}$, we find that anthropomorphised environmental feedback may be most effective both in inspiring action at work and at home. However, we also found that people can have different motivations across work and home contexts (e.g., identifying with the organisations' values vs. saving money), and such psychological factors need to be better understood while designing interventions that further catalyse contextual spillovers.

The study has also several limitations. Like in many other studies on food consumption and food waste, directly observed individual-level data on food wasted at work and at home was not available. We also do not have a control group which received no feedback at all, so are unable to shed light on whether sites would have shown a more rapid and steep increasing trend in the absence of any feedback, or indeed if there is any contextual spillover from any feedback. Lastly, this study was conducted during a period where pandemic controls were lifting, leading to some uncertainty about how representative and generalizable these results are at other times. Future studies can address these issues, as well as examine the impact of such campaigns in conjunction with other nudges like reducing plate size. Despite these concerns, this study takes the first step towards measuring food waste reductions in the hospitality sector using a mix of technological and behavioural interventions, and the results encouragingly suggest that food waste reduction efforts at work can also help at home.

\section{Methods}

For the field study, we used a difference-in-difference (DID) regression to explore if the environmental campaign affected food waste behaviours at work:

grams_per_cover $r_{\text {it }}$

$$
\begin{aligned}
& =\alpha_{i}+\beta_{1}{\text { treatment } B_{i} \times \text { during_campaig }_{t}} \\
& +\beta_{2} \text { treatment }_{i} \times \text { during_campaign } n_{t}+\beta_{3}{\text { treatment } B_{i}} \times \text { after_campaign } n_{t} \\
& +\beta_{4} \text { treatment }_{i} \times \text { after_campaig } n_{t}+\gamma_{t}+\varepsilon_{i t}
\end{aligned}
$$

$\boldsymbol{\alpha}_{\boldsymbol{i}}$ denotes the site fixed effects.

$\gamma_{t}$ denotes the time-varying variables, including 2 dummies for during_campaign and after_campaign, 6 dummies for the days of the week (Sunday omitted) and 5 monthly dummies (Jan to May 2021, Dec 2020 omitted). 


\section{$\boldsymbol{\beta}_{1}$ and $\boldsymbol{\beta}_{2}$}

are the DID estimators of the treatment effects of the two types of environmental messages in sites B and C, respectively, during the food waste campaign.

\section{$\boldsymbol{\beta}_{3}$ and $\boldsymbol{\beta}_{4}$} estimate if the treatment effects carryover after the campaign ended in sites B and C, respectively

\section{$\boldsymbol{\beta}_{1}-\boldsymbol{\beta}_{4}$}

were all significantly lower than zero (see SI Table S2), suggesting that the treatments in sites $B$ and $C$ were associated with a reduction in food waste and the effects lingered after the campaign. Nevertheless, there are some caveats. We assume that the treatment and control sites should have the same, parallel trends in food waste behaviours in the absence of any treatments. While this is often verified by showing that pre-treatment trends are parallel for a reasonable timeframe, unfortunately we do not have a long time series of data to do so in our case: this is because the WS system was installed in the control site one month later than the two treatment sites, and because the sensitivity of the WS system was calibrated and in adjustment during that month. So, we only had 3 weeks of reliable data from all the sites before the treatment, during which the parallel trend assumption was satisfied. Furthermore, since we could only access three hotel canteens for staff, we could only allocate one site to each treatment, and therefore we could not employ a clustered randomised design nor have enough degrees of freedom to be able to cluster standard errors at the site level in the regressions.

In the survey study, we conducted a multiple linear regression to examine the spillovers from work to home:

\section{$E H=\alpha+\beta_{1} E W 1+\beta_{2} E W 2+\beta_{3}$ treatment_siteB $+\beta_{4}$ treatment_siteC}

\section{$+\gamma_{1}$ environmental_beliefs $+\gamma_{2}$ motivations $+\gamma_{3}$ demographics $+\varepsilon$}

Efforts to reduce food waste at work were measured by asking if the respondent had done any of the following during the study period: "Had a conversation with work colleagues about food waste" and "Tried to reduce food waste at work" ( $1=$ "Never" to $5=$ "Always"). The two items were not highly correlated $(r=0.33)$ and were thus analysed separately (i.e., EW1 and EW2). Efforts to reduce food waste at home were measured by asking if the respondent had done any of the following during the study period: "Had a conversation with friends/family about food waste" "Tried to reduce food waste at home" "Threw away less food" "Used leftover food for meals or cooking" ( $1=$ "Never" to 5 = "Always"). The reliability of this scale was satisfactory (Cronbach's $a=0.68$ ), so we used the composite variable (i.e., EH, the mean of the four items) as our main outcome and replicated the analysis on the four items separately. Both items for efforts at work were positively associated with efforts to reduce food waste at home and the effects were statistically significant. Additional analyses on the four items for efforts at home yielded similar results (see SI Table S4). 


\section{Declarations}

\section{Acknowledgements}

We would like to thank Sasha Maisel, Amy Hong, Emily Comyn, and Marc Zornes from Winnow, and Denise Chen, Sylvie Fung, Hannah Chung, Sammy Wong, Perry Yuen, Peter Tong, Tiffany Lu, Joyce Kuang, and Andrew Kok from Melco. We would also like to thank Julien Picard for comments.

\section{Author contributions}

GS, FW, MMG, SM conceptualized the study, designed the methodology, FW and GS analysed the data and interpreted the results, FW curated and managed the data, GS and FW wrote the original draft, MMG and SM contributed to subsequent versions. GS supervised the project. All the feedback interventions were co-designed with Winnow and the project was jointly implemented by Winnow, Melco and LSE.

\section{Competing Interests statement}

None to declare.

\section{References}

1. West, P. C. et al. Leverage points for improving global food security and the environment. Science 345, 325-328 (2014).

2. Mehrabi, Z. Food system collapse. Nature Climate Change 10, 16-17 (2020).

3. Reisch, L. A. et al. Mitigating climate change via food consumption and food waste: A systematic map of behavioral interventions. Journal of Cleaner Production 123717 (2020).

4. Garnett, E. E., Marteau, T. M., Sandbrook, C., Pilling, M. A. \& Balmford, A. Order of meals at the counter and distance between options affect student cafeteria vegetarian sales. Nat Food 1, 485-488 (2020).

5. Lim, V., Bartram, L., Funk, M. \& Rauterberg, M. Eco-Feedback for Food Waste Reduction in a Student Residence. Front. Sustain. Food Syst. 5, (2021).

6. Reynolds, C. et al. Review: Consumption-stage food waste reduction interventions - What works and how to design better interventions. Food Policy 83, 7-27 (2019).

7. Verfuerth, C., Jones, C. R., Gregory-Smith, D. \& Oates, C. Understanding Contextual Spillover: Using Identity Process Theory as a Lens for Analyzing Behavioral Responses to a Workplace Dietary Choice Intervention. Front. Psychol. 10, (2019).

8. Maki, A. et al. Meta-analysis of pro-environmental behaviour spillover. Nature Sustainability 2, 307 (2019). 
9. Camilleri, A. R., Larrick, R. P., Hossain, S. \& Patino-Echeverri, D. Consumers underestimate the emissions associated with food but are aided by labels. Nature Climate Change 9, 53-58 (2019).

10. Gil, J. Going to waste. Nat Food 1, 192-192 (2020).

11. Wolstenholme, E., Poortinga, W. \& Whitmarsh, L. Two Birds, One Stone: The Effectiveness of Health and Environmental Messages to Reduce Meat Consumption and Encourage Pro-environmental Behavioral Spillover. Front. Psychol. 11, (2020).

12. Whitmarsh, L. \& O'Neill, S. Green identity, green living? The role of pro-environmental self-identity in determining consistency across diverse pro-environmental behaviours. Journal of Environmental Psychology 30, 305-314 (2010).

13. Capstick, S., Whitmarsh, L., Nash, N., Haggar, P. \& Lord, J. Compensatory and Catalyzing Beliefs: Their Relationship to Pro-environmental Behavior and Behavioral Spillover in Seven Countries. Front. Psychol. 10, (2019).

14. Asensio, O. I. \& Delmas, M. A. Nonprice incentives and energy conservation. PNAS 112, E510-E515 (2015).

15. Nomura, H., John, P. C. \& Cotterill, S. The use of feedback to enhance environmental outcomes: a randomised controlled trial of a food waste scheme. Local Environment 16, 637-653 (2011).

16. Shao, X., Jeong, E., Jang, S. S. \& Xu, Y. Mr. Potato Head fights food waste: The effect of anthropomorphism in promoting ugly food. International Journal of Hospitality Management 89 , 102521 (2020).

17. Cooremans, K. \& Geuens, M. Same but Different: Using Anthropomorphism in the Battle Against Food Waste. Journal of Public Policy \& Marketing 38, 232-245 (2019).

18. Ahn, H.-K., Kim, H. J. \& Aggarwal, P. Helping fellow beings: Anthropomorphized social causes and the role of anticipatory guilt. Psychological Science 25, 224-229 (2014).

19. Hebrok, M. \& Boks, C. Household food waste: Drivers and potential intervention points for design An extensive review. Journal of Cleaner Production 151, 380-392 (2017).

20. Thyberg, K. L. \& Tonjes, D. J. Drivers of food waste and their implications for sustainable policy development. Resources, Conservation and Recycling 106, 110-123 (2016).

21. Visschers, V. H. M., Wickli, N. \& Siegrist, M. Sorting out food waste behaviour: A survey on the motivators and barriers of self-reported amounts of food waste in households. Journal of Environmental Psychology 45, 66-78 (2016).

\section{Figures}




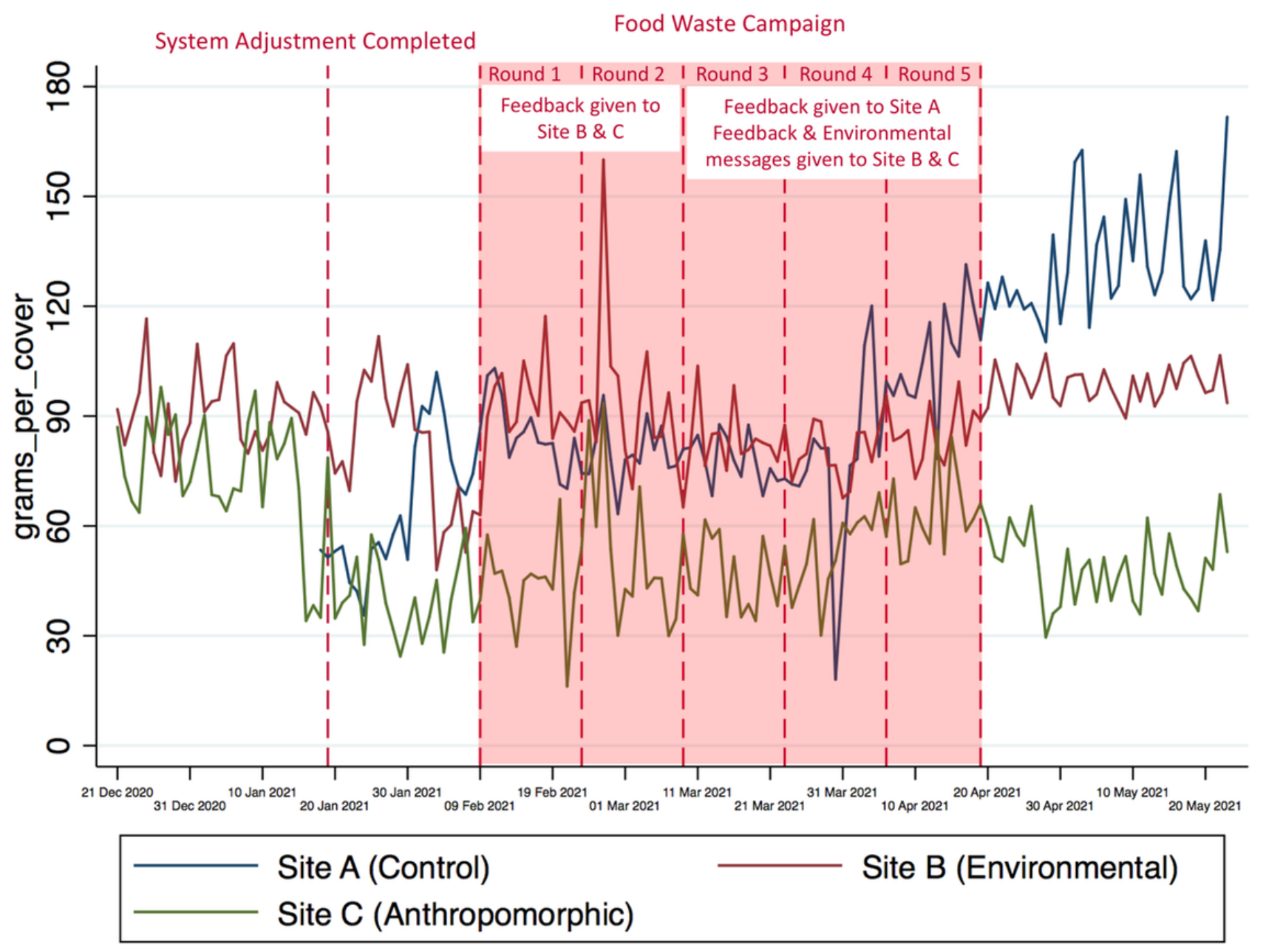

Figure 1

Average food waste per meal across sites (in grams) 

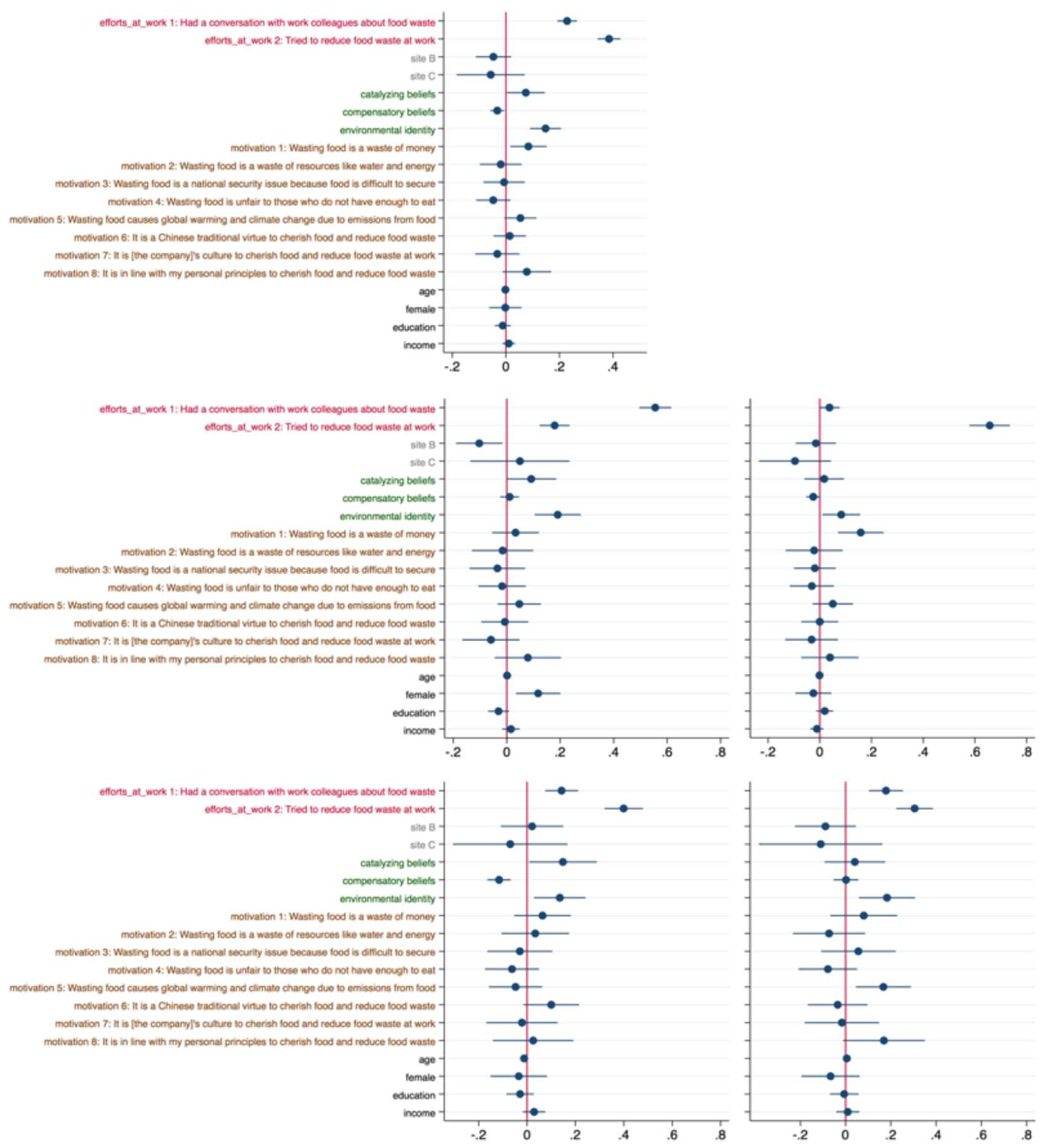

Figure 2

Regression models testing spillovers from work to home. The five panels show the estimated coefficients and their $95 \%$ confidence intervals based on our OLS regression models using either the composite variable for efforts at home, or the four items, respectively, as the dependent variable. The regression coefficients are significant at the $5 \%$ level when their $95 \%$ confidence intervals do not cross the red line.

\section{Supplementary Files}


This is a list of supplementary files associated with this preprint. Click to download.

- Sl.docx 